\title{
Avaliação de uma ferramenta nutricional como preditor de prognóstico do paciente gravemente enfermo
}

\section{Evaluation of a nutritional tool as a predictor of the prognosis of the critically ill patient}

\author{
Natália Linhares Ponte Aragão ${ }^{1}$. Ana Cecília Santos Martins Cláudio Mourão². Beatriz Amorim Beltrão ${ }^{3}$. \\ Vitor Nogueira Araújo ${ }^{4}$. Antônio Brazil Viana Júnior ${ }^{5}$. Arnaldo Aires Peixoto Junior ${ }^{6}$. \\ 1 Médica residente do Programa de Medicina Intensiva em Nutrição Enteral e Parenteral, Universidade Federal do Ceará (UFC), \\ Fortaleza, Ceará, Brasil. 2 Médica intensivista da Unidade de Terapia Intensiva, Hospital Universitário Walter Cantídio (HUWC), \\ Fortaleza, Ceará, Brasil. 3 Enfermeira da Unidade de Terapia Intensiva, Hospital Universitário Walter Cantídio (HUWC), \\ Fortaleza, Ceará, Brasil. 4 Médico intensivista da Unidade de Terapia Intensiva, Hospital Universitário Walter Cantídio (HUWC), \\ Fortaleza, Ceará, Brasil. 5 Estatístico da Unidade de Pesquisa Clínica, Hospital Universitário Walter Cantídio (HUWC), Fortaleza, \\ Ceará, Brasil. 6 Doutorado em Farmacologia, Programa de Residência em Medicina Intensiva, Programa de Residência em \\ Medicina Intensiva em Nutrição Enteral e Prenteral, Professor do Departamento de Medicina Clínica, Faculdade de Medicina da \\ Universidade Federal do Ceará (UFC), Fortaleza, Ceará, Brasil.
}

\section{RESUMO}

Objetivos: avaliar o escore de risco nutricional NUTRIC como preditor prognóstico e compará-lo com outros escores de gravidade. Método: estudo observacional, retrospectivo, com avaliação dos registros de 80 pacientes internados em uma unidade de terapia intensiva adulto, de março a junho de 2017. Foi estimado o risco nutricional através do escore NUTRIC. Resultados: foram construídas curvas ROC (Receiver Operating Characteristic): as áreas sob a curva do escore APACHE II e SOFA no primeiro e no terceiro dia foram de 0,$711 ; 0,701$ e 0,809 , respectivamente, e a do NUTRIC de 0,713 . Foi encontrado um ponto de corte do NUTRIC de 5, com acurácia de 73,6\% (IC (intervalo de confiança) 95\%: 0,628-0,844). Os pacientes foram divididos em 2 subgrupos: G1, com pontuação $\geq 5$ e G2 com pontuação $<5$. O subgrupo G1 apresentou mais disfunções orgânicas, mensuradas pelo SOFA do terceiro dia $(8,8 \pm 4,8 \times 4,2 \pm 4,3 ; \mathrm{p}<0,001)$ e maior taxa de mortalidade na UTI $(66,0 \times 26,7 \%$; $\mathrm{p}=0,002)$. Conclusão: o escore NUTRIC pode predizer um desfecho desfavorável, semelhante aos escores APACHE II e o SOFA. Parece ser útil em prever a ocorrência de novas disfunções e mortalidade.

Palavras-chave: Avaliação nutricional. Prognóstico. Unidade de Terapia Intensiva. Mortalidade. Índice de gravidade de doença.

\section{ABSTRACT}

Objectives: To evaluate the NUTRIC nutritional risk score as a predictor of prognosis and to compare it with other severity scores. Method: an observational, retrospective study with the evaluation of the records of 80 patients hospitalized in an adult intensive care unit from March to June 2017. The nutritional risk was estimated through the NUTRIC score. Results: Receiver Operating Characteristic (ROC) curves were constructed: the areas under the curve of the APACHE II and SOFA score on the first and third day were $0.711 ; 0.701$ and 0.809 , respectively, and that of the NUTRIC of 0.713 . A NUTRIC cut-off point of 5 was found, with accuracy of 73.6\% (95\% CI: 0.628-0.844). Patients were divided into 2 subgroups: G1, with score $\geq 5$ and G2 with score $<5$. The G1 subgroup presented more organ dysfunctions, measured by the SOFA on the third day $(8.8 \pm 4.8 \times 4.2 \pm 4,3 ; \mathrm{p}<0.001)$ and higher ICU mortality rate $(66.0 \times 26.7 \%, \mathrm{p}=0.002)$. Conclusion: the NUTRIC score can predict an unfavorable outcome, similar to APACHE II and SOFA scores. It seems to be useful in predicting the occurrence of new malfunctions and mortality.

Keywords: Nutrition Assessment. Prognosis. Intensive Care Units. Mortality. Severity of illness index.

Autor correspondente: Natália Linhares Ponte Aragão, Rua Capitão Francisco Pedro, 1290, Rodolfo Teófilo, Fortaleza, Ceará. CEP: $60430-370$. Telefone: +55 85 3366-8162. E-mail: nat_Ipa@hotmail.com

Conflito de interesses: Não há qualquer conflito de interesses por parte de qualquer um dos autores.

Recebido em: 09 Fev 2018; Revisado em: 29 Jul 2018; Aceito em: 29 Jul 2018. 


\section{INTRODUÇÃO}

A incidência de desnutrição no ambiente hospitalar é alta, com relatos de variação de 20-69\%. No entanto, a prevalência dentro da unidade de terapia intensiva (UTI) é consideravelmente maior, $40-100 \%{ }^{1}$ A desnutrição do paciente crítico está associada com piores desfechos, como prejuízo da cicatrização, aumento das taxas de infecção e da mortalidade. ${ }^{2}$

O estado nutricional na UTI não é influenciado apenas pelo jejum, mas pela severidade do processo fisiopatológico da doença que levou o paciente a ser admitido. ${ }^{3}$ Durante a resposta ao estresse, ocorre hipermetabolismo, que inclui aumento do gasto energético basal, do consumo de oxigênio, do débito cardíaco e do consumo de nutrientes nobres como proteínas sendo utilizadas como substrato energético. ${ }^{4}$

Um consenso da Sociedade Americana de Nutrição Enteral e Parenteral (ASPEN) reconheceu a importância da inflamação na caracterização da desnutrição e recomendou uma definição baseada na presença de pelo menos duas das seguintes características: aporte energético insuficiente, perda de peso, perda de massa magra, perda de gordura subcutânea, acúmulo de líquidos localizado ou generalizado, redução da funcionalidade no contexto de lesão aguda ou doença crônica e inanição relacionada à desnutrição. No entanto, não há consenso quanto a melhor ferramenta para identificar esses pacientes, sobretudo na UTI. ${ }^{5}$

Muitas ferramentas tradicionais não fornecem informações adequadas do estado inflamatório. O escore Nutrition Risk in Critically ill (NUTRIC), que foi introduzido por Heyland et al., tem o objetivo de identificar os pacientes críticos que mais se beneficiam de terapia nutricional agressiva, relacionando jejum, inflamação e desfechos. ${ }^{6}$

O objetivo do nosso estudo foi avaliar o escore de risco nutricional NUTRIC modificado como preditor de piores desfechos na UTI e comparar este escore com outros escores de gravidade que estimam mortalidade e morbidade.

\section{MATERIAL E MÉTODOS}

Trata-se de estudo retrospectivo observacional, no qual foram analisados dados obtidos nos prontuários dos pacientes internados em uma UTI clínica adulto do Hospital Universitário Walter Cantídio da Universidade Federal do Ceará.

Foram avaliados todos os pacientes adultos, internados consecutivamente, no período de março a junho de 2017. Foram colhidos dados demográficos, escores de gravidade Acute Physiology and Chronic Health Evaluation II (APACHE II) ${ }^{7}$ e Sequential Organ Failure Assessment (SOFA). ${ }^{8}$

Foi estimado o risco nutricional através da utilização do escore NUTRIC. O escore NUTRIC combina parâmetros de jejum agudo (duração do internamento antes da UTI), com parâmetros inflamatórios agudos (interleucina 6) e crônicos (comorbidades) e severidade da doença (escores de gravidade), descritos no Quadro 1. Como a interleucina 6 não é disponível na maioria das UTIs, foi validada a utilização do escore NUTRIC modificado, sem a mesma, conforme o Quadro 2. ${ }^{6}$ Foi realizada a determinação de um ponto de corte do escore como preditor de mortalidade através da utilização da curva ROC. Os pacientes foram divididos em 2 subgrupos: G1, composto por pacientes com escore NUTRIC alto $(\geq 5$ pontos), e subgrupo G2, formado por pacientes com escore NUTRIC baixo ( $<5$ pontos).

Quadro 1. Variáveis do escore NUTRIC.

\begin{tabular}{|l|c|c|}
\hline Variáveis & Variação & Pontos \\
\hline Idade & $<50$ & 0 \\
\hline & $50 \leq 75$ & 1 \\
\hline APACHE II & $>75$ & \\
\hline & $<15$ & 1 \\
\hline & $15 \leq 20$ & 2 \\
\hline & $20-28$ & 3 \\
\hline SOFA & $>28$ & 0 \\
\hline & $<6$ & 1 \\
\hline & $6 \leq 10$ & 1 \\
\hline Número de comorbidades & $>10$ & 2 \\
\hline & $>-1$ & 0 \\
\hline $\begin{array}{l}\text { Dias no hospital antes da } \\
\text { admissão na UTI }\end{array}$ & $0 \leq 1$ & 26,7 \\
\hline & $>1$ & \\
\hline Interleucina 6* & 66,0 & \\
\hline
\end{tabular}

Nota: *é aceitável não incluir interleucina 6 no cálculo quando não disponível.

Fonte: adaptada de: Heyland DK, Dhaliwal R, Jiang X, Day AG. Identifying critically ill patients who benefit the most from nutrition therapy: the development and initial validation of a novel risk assessment tool. Crit Care. 2011;15(6):R268.

Quadro 2. Pontuação do escore NUTRIC modificado (interleucina não disponível).

\begin{tabular}{|l|l|l|}
\hline $\begin{array}{c}\text { Somatório } \\
\text { de pontos }\end{array}$ & Categoria & \multicolumn{1}{|c|}{ Explicação } \\
\hline $\mathbf{5 - 9}$ & Escore Alto & $\begin{array}{l}\text { Associação com piores desfechos } \\
\text { clínicos. Esses pacientes se } \\
\text { beneficiam de terapia nutricional } \\
\text { mais agressiva. }\end{array}$ \\
\hline $\mathbf{0 - 4}$ & Escore Baixo & $\begin{array}{l}\text { Esses pacientes têm baixo risco } \\
\text { de desnutrição. }\end{array}$ \\
\hline
\end{tabular}


As variáveis categóricas foram descritas em frequência absoluta e relativa (percentual). Variáveis contínuas com distribuição normal foram expressas em média e desvio padrão, e as com distribuição não-normal em mediana e percentis. A comparação entre os dois subgrupos foi realizada pelo teste qui-quadrado de Pearson ou teste exato de Fisher para as variáveis categóricas; e pelo teste $\mathrm{t}$ de Student ou teste de Mann-Whitney para variáveis numéricas, conforme a normalidade dos dados. Foram construídas curvas Receiver Operating Characteristic (ROC) para avaliar e comparar a capacacidade preditiva dos marcadores de gravidade. Os dados foram tabulados no software Microsoft Excel 2007 ${ }^{\circledR}$ para Windows ${ }^{\circledR}$ e as análises foram realizadas por meio do software SPSS Statistics ${ }^{\circledR}$, versão 20.0.0.

Este estudo faz parte de uma coorte retrospectiva, com coleta de dados registrados em prontuários, com dispensa do preenchimento de Termo de Consentimento Livre e Esclarecido. Foi aprovado pelo Comitê de Ética em Pesquisa da instituição (CAAE: 64529817.1.0000.5045), estando de acordo com a resolução 466/12 do Conselho Nacional de Saúde e a Declaração de Helsinque.

\section{RESULTADOS}

No período estudado, 80 pacientes foram admitidos na UTI. Estes tinham uma média de idade de 56,5 $\pm 9,2$ anos. A maioria dos indivíduos eram do sexo masculino $(53,7 \%)$. Como marcador de gravidade, o APACHE II médio foi de $20,0 \pm 6,6$ pontos, o SOFA à admissão de $8,4 \pm 4,6$ pontos e o SOFA no terceiro dia de internamento de $6,9 \pm 5,1$ pontos. A

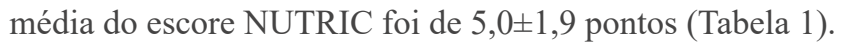

Foram construídas curvas ROC no intuito de comparar os escores de gravidade (Gráfico 1), verificando qual seria o melhor marcador de gravidade. A área sob a curva do escore APACHE II e SOFA no primeiro e no terceiro dia foram de 0,$711 ; 0,701$ e 0,809 , respectivamente. Já a área sob a curva do escore NUTRIC foi de 0,713 . Quanto à predição de óbito, não houve diferença entre os escores NUTRIC e APACHE II ( $p=0,76)$; NUTRIC e SOFA na admissão $(p=0,83)$; e NUTRIC e SOFA no terceiro dia de UTI $(\mathrm{p}=0,09)$.

Tabela 1. Caracterização dos pacientes estudados $(\mathrm{N}=80)$.

\begin{tabular}{lc}
\hline Características & Valores \\
\hline Dados demográficos & \\
Idade (anos)* & $56,51 \pm 9,2$ \\
Sexo masculino** & 53,7 \\
Marcadores de gravidade* & \\
Escore APACHE II & $20,0 \pm 6,6$ \\
Escore SOFA D1 & $8,4 \pm 4,6$ \\
Escore SOFA D3 & $6,9 \pm 5,1$ \\
Escore NUTRIC & $5,0 \pm 1,9$ \\
\hline
\end{tabular}

Nota: * média \pm desvio padrão; ** N (\%).
Gráfico 1. Curva ROC: comparação entre os escores APACHE II, SOFA D1, SOFA D3 e escore NUTRIC como preditores de mortalidade.

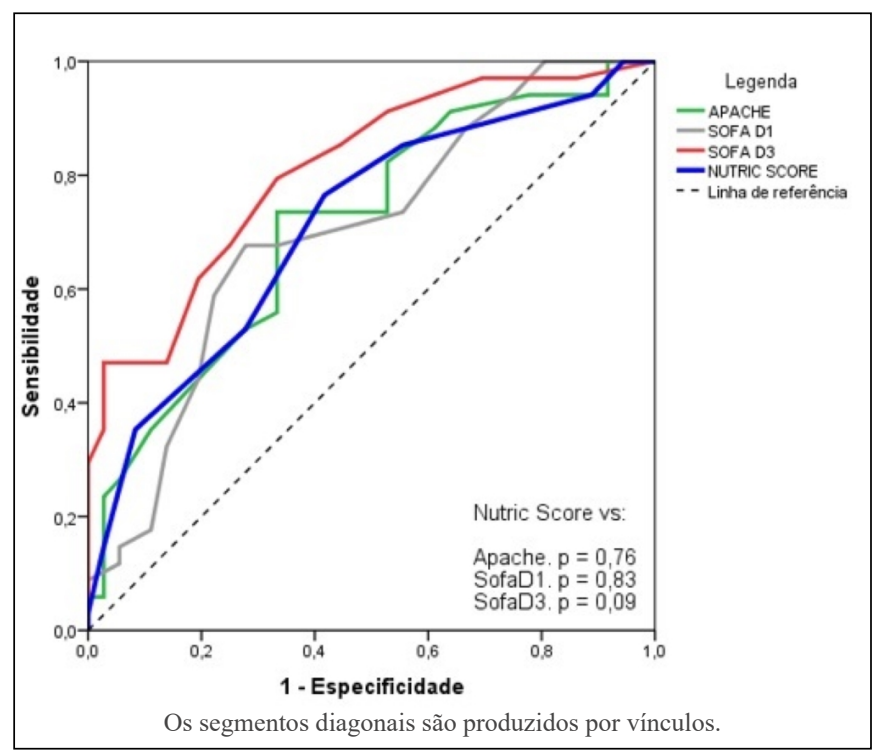

Foi possível o encontro de um ponto de corte do NUTRIC de 5 pontos, que permitiu identificar o risco de óbito com uma sensibilidade de $80,4 \%$, especificidade de $56,4 \%$ e acurácia de 73,6\% (IC (intervalo de confiança) 95\%: 62,8-84,4).

Os pacientes foram divididos em 2 subgrupos de acordo com o ponto de corte de 5 , de modo que fizeram parte do subgrupo G1 aqueles com pontuação maior ou igual a 5 (NUTRIC alto) e foram alocados no subgrupo G2 os que tiveram pontuação inferior a 5 .

A comparação entre os 2 subgrupos está descrita na Tabela 2. A maioria dos pacientes foram alocados no subgrupo G1 (62,5\%). Esse subgrupo apresentou um maior grau de disfunções orgânicas ao longo do internamento, mensurada pelo SOFA no terceiro dia na UTI $(8,8 \pm 4,8 \mathrm{vs}$. 4,2 $\pm 4,3$ pontos; $\mathrm{p}<0,001)$, e maior taxa de mortalidade na UTI ( 66,0 vs. $26,7 \%$; $\mathrm{p}=0,002)$.

Tabela 2. Comparação entre os subgrupos G1 e G2.

\begin{tabular}{lccc}
\hline Variáveis & G1 & G2 & $p$ \\
\hline Escore SOFA D3* & $(\mathrm{N}=50)$ & $(\mathrm{N}=30)$ & \\
Traqueostomia** & $8,8 \pm 4,8$ & $4,2 \pm 4,3$ & $\mathrm{p}<0,001$ \\
Cuidados paliativos** & 36,0 & 26,7 & 0,38 \\
Permanência na UTI (dias)* & $11,2 \pm 10,1$ & $9,8 \pm 11,6$ & 0,56 \\
Permanência hospitalar & $9,0 \pm 7,4$ & $9,5 \pm 6,5$ & 0,83 \\
após UTI (dias)* & & & 0,062 \\
Mortalidade** & 66,0 & 26,7 & 0,002 \\
\hline
\end{tabular}

Nota: * média \pm desvio padrão; ** N (\%). 
Entretanto, não houve diferença quanto a tempo de permanência na UTI $(11,2 \pm 10,1$ vs. $9,8 \pm 11,6$ dias; $\mathrm{p}=0,56)$; tempo de permanência hospitalar após a alta da UTI $(9,0 \pm 7,4$ vs. 9,5 $\pm 6,5$ dias; $\mathrm{p}=0,83)$; necessidade de traqueostomia para desmame de ventilação mecânica prolongada (36,0 vs. 26,7\%; $\mathrm{p}=0,38)$ ou de acompanhamento pelo serviço de cuidados paliativos $(32,0$ vs. $13,3 \% ; \mathrm{p}=0,062)$.

\section{DISCUSSÃO}

Após uma agressão grave, ocorrem várias alterações metabólicas e hormonais que convergem para a manutenção da homeostase e da hemodinâmica do organismo. Tais alterações provocam, dentre outros efeitos, aumento da resistência à insulina e do catabolismo proteico. Assim, em decorrência do elevado estado metabólico, todos os pacientes críticos deveriam ser considerados de elevado risco nutricional. ${ }^{9}$

Nesse estudo observacional e retrospectivo, 62,5\% dos pacientes admitidos à UTI eram de risco nutricional (de acordo com o escore NUTRIC). A prevalência de desnutrição na UTI varia de acordo com a ferramenta utilizada para a identificação, podendo chegar a 100\%. ${ }^{1}$ Kalaselvan et al, que estudaram apenas pacientes sob ventilação mecânica, encontraram um valor inferior ao nosso: $48,6 \%$ dos pacientes admitidos tinham um escore NUTRIC igual ou superior a $5 .{ }^{10}$ A média do escore NUTRIC do trabalho de Kalaiselvan foi 4 , inferior à média encontrada no estudo original de Heyland et al, que inicialmente validou o escore. ${ }^{6}$ A média do nosso escore foi $5,0 \pm 1,9$. Isso pode ser decorrente dos nossos maiores escores de disfunções orgânicas à admissão. O trabalho de Kalaiselvan tinha uma média de SOFA no D1 de 6.7, o de Heyland, de 7 , e o nosso de $8,4 \pm 4,6 .^{10,6}$

Nesse trabalho foi encontrada uma habilidade preditiva do NUTRIC de se relacionar com mortalidade através do ponto de corte de 5,0 (AUC (area under the ROC curve): 0,736; IC 95\%: 0,628-0,844). O estudo de Heyland et al encontrou valor semelhante (AUC: 0,783) com o mesmo ponto de corte, analisando mortalidade de 28 dias. ${ }^{6}$ Outros estudos de validação do NUTRIC também avaliaram essa relação com mortalidade. Rahman et al estudaram a população caucasiana (AUC: 0,768) e Mukhopadhyay estudou a asiática (AUC: 0,701). Esses estudos relataram uma melhora da mortalidade de 28 dias com alta taxa de adequação nutricional. ${ }^{11,12}$

No nosso trabalho não foi possível encontrar relação de valor do escore NUTRIC com aumento do tempo de permanência. Higgins et al demonstraram que pacientes com escore mais alto tinham maior permanência. Também relataram que uma acurada identificação desses pacientes permitiu o fornecimento

\section{REFERÊNCIAS}

1. Caporossi FS, Caporossi C, Dock-Nascimento D, AguilarNascimento JE. Measurement of the thickness of the adductor pollicis muscle as a predictor of outcome in critically ill patients. Nutr Hosp. 2012;27(2):490-5.

2. Villet S, Chiolero RL, Bollmann MD, Revelly JP, Cayeux de uma terapia nutricional mais adequada, com redução da permanência com essa estratégia. ${ }^{13}$ Mukhopadhyay et al também associaram o escore com aumento da permanência, mostrando uma redução dos custos hospitalares com a adequação nutricional. ${ }^{12}$

Encontramos que os pacientes com escore elevado também tinham mais disfunções novas durante o internamento na UTI através do aumento do SOFA no terceiro dia. O trabalho de Özbilgin et al mostrou que os pacientes com NUTRIC alto tinham mais complicações durante o internamento. Descreveram maior ocorrência de pneumonia, fibrilação atrial, delirium, insuficiência renal, uso de inotrópicos e aumento do tempo de ventilação mecânica em pacientes cirúrgicos. ${ }^{14}$

A definição de risco nutricional sofreu adaptações a partir do contexto de desnutrição relacionada a doença, de modo que, pode envolver variáveis tradicionais como índice de massa corpórea, perda ponderal e redução na ingesta de nutrientes, mas também deve incluir fatores relacionados à gravidade da doença, como APACHE II e SOFA. ${ }^{6}$ No nosso trabalho, fizemos uma comparação desses escores, com a análise de curvas ROC. O escore mais acurado foi o SOFA no terceiro dia (AUC: 0,809), que já descreve a evolução do paciente. No entanto, considerando apenas os escores calculados à admissão, o NUTRIC apresentou maior capacidade de predizer o óbito (AUC: 0,713) que o APACHE II e o SOFA isoladamente (AUC: 0,711 e 0,701).

Esse estudo tem limitações. Primeiro, foi um estudo retrospectivo realizado em um único centro. Além disso, com poucos dados de adequação nutricional, não se pode avaliar os efeitos da terapia nutricional na redução da mortalidade e das complicações durante o internamento na UTI.

Apesar dessas limitações, conseguimos mostrar uma grande prevalência de desnutrição em uma UTI brasileira, com consequências graves, como aumento da mortalidade e do número de disfunções durante o internamento. Esse estudo alerta para a necessidade de uma adequada avaliação do risco nutricional, de modo a otimizar a terapia nutricional e tentar melhorar desfechos clínicos.

\section{CONCLUSÃO}

O escore de risco nutricional NUTRIC, originalmente utilizado para identificar pacientes que se beneficiam de terapia nutricional precoce, pode predizer um desfecho desfavorável, semelhante aos escores APACHE II e o SOFA. Parece ser útil em predizer a ocorrência de novas disfunções e mortalidade.

MC, Delarue J, et al. Negative impact of hypocaloric feeding anenergy balance on clinical outcome in ICU patients. Clin Nutr. 2005;24(4):502-9.

3. Kondrup J. Nutritional risk scoring systems in the intensive care unit. Curr Opin Clin Nutr Metab Care. 2014;17(2):177-82. 
4. Hill AG, Hill GL. Metabolic responde to severe injury. Br J Surg. 1998;85(7):884-90.

5. White JV, Guenter P, Jensen G, Malone A, Schofield M. Consensus statement of the Academy of Nutrition and Dietetics/American Society for Parenteral and Enteral Nutrition: characteristics recommended for the identification and documentation of adult malnutrition (undernutrition). J Acad Nutr Diet. 2012;112(5):730$730-8$.

6. Heyland DK, Dhaliwal R, Jiang X, Day AG. Identifying critically ill patients who benefit the most from nutrition therapy: the development and initial validation of a novel risk assessment tool. Crit Care. 2011;15(6):R268.

7. Knaus WA, Draper EA, Wagner DP, Zimmerman JE. APACHE II: a severity of disease classification system. Crit Care Med. 1985;13(10):818-29.

8. Vincent JL, Moreno R, Takala J, Willatts S, De Mendonça A, Bruining $\mathrm{H}$, et al. The SOFA (Sepsis-related Organ Failure Assessment) score to describe organ dysfunction/failure. On behalf of the Working Group on Sepsis-Related Problems of the European Society of Intensive Care Medicine. Intensive Care Med. 1996;22(7):707-10.
9. Jensen GL, Wheeler D. A new approach to defining and diagnosing amlnutrition in adult critcal illness. Curr Opin Crit Care. 2012;18(2):206-11.

10. Kalaiselvan MS, Renuka1 MK, Arunkumar AS. Use of Nutrition Risk in Critically ill (NUTRIC) score to assess nutritional risk in mechanically ventilated patients: a prospective observational study. Indian J Crit Care Med. 2017;21(5):253-6.

11. Rahman A, Hasan R, Agarwala R, Martin C, Day A, Heyland D. Identifying critically-ill patients who will benefit most from nutritional therapy: further validation of the "modified NUTRIC" nutritional risk assessment tool. Clin Nutr. 2016;35:158-62.

12. Mukhopadhyay A, Henry J, Ong V, Leong CS, Teh A, van Dam RM, et al. Association of modified NUTRIC score with 28-day mortality in critically ill patients. Clin Nutr. 2017;36(4):1143-8.

13. Higgins TL, McGee WT, Steingrub JS, Rapoport J, Lemeshow $\mathrm{S}$, Teres D. Early indicators of prolonged intensive care unit stay: impact of illnessseverity, physician staffing, and pre-intensive care unit length of stay. Crit Care Med. 2003;31(1):45-51.

14. Özbilgin S, Hanc1 V, Ömür D, Özbilgin M, Tosun M, Yurtlu S, et al. Morbidity and mortality predictivity of nutritional assessment tools in the postoperative care unit. Medicine (Baltimore). 2016;95(40):e5038.

\section{Como citar:}

Aragão NL, Mourão AC, Beltrão BA, Araújo VN, Viana AB Júnior, Peixoto AA Junior. Avaliação de uma ferramenta nutricional como preditor de prognóstico do paciente gravemente enfermo. Rev Med UFC. 2018 jan-mar;59(1):34-38. 